Masaya Kawamura*

\title{
Estimates for a function on almost Hermitian manifolds
}

https://doi.org/10.1515/coma-2020-0118

Received December 15, 2020; accepted August 23, 2021

Abstract: We study some estimates for a real-valued smooth function $\varphi$ on almost Hermitian manifolds. In the present paper, we show that $\partial \partial \bar{\partial} \varphi$ and $\bar{\partial} \partial \bar{\partial} \varphi$ can be estimated by the gradient of the function $\varphi$.

Keywords: almost Hermitian metric, almost complex manifold, Chern connection

MSC: $32 \mathrm{Q} 60$ (primary); 53C15; 53C55 (secondary)

\section{Introduction}

Let $\left(M^{2 n}, J\right)$ be an almost complex manifold of real dimension $2 n$ and $g$ an almost Hermitian metric on $M$. Let $\left\{Z_{r}\right\}$ be an arbitrary local $(1,0)$-frame around a fixed point $p \in M$. We shall use the following notations: for a function $\varphi, \nabla_{i} \nabla_{\bar{j}} \varphi:=\nabla_{Z_{i}} \nabla_{Z_{j}} \varphi$ and

$$
\varphi_{i \bar{j}}:=\partial_{i} \partial_{\bar{j}} \varphi=\nabla_{i} \nabla_{\bar{j}} \varphi,
$$

where $\nabla$ is the Chern connection with respect to $g, \partial_{i} \partial_{\bar{j}} \varphi=\left(Z_{i} Z_{\bar{j}}-\left[Z_{i}, Z_{\bar{j}}\right]^{(0,1)}\right) \varphi, \nabla_{i} \nabla_{\bar{j}} \varphi=Z_{i} Z_{\bar{j}}(\varphi)-B_{i \bar{j}}^{\bar{s}} Z_{\bar{s}}(\varphi)$ (see Section 2 for the definition of $\left.B_{i j}^{\bar{s}}\right)$. Since we have $\left[Z_{i}, Z_{\bar{j}}\right]^{(0,1)}(\varphi)=B_{i j}^{\bar{s}} Z_{\bar{s}}(\varphi)$, we obtain that $\partial_{i} \partial_{\bar{j}} \varphi=\nabla_{i} \nabla_{\bar{j}} \varphi$.

Theorem 1.1. Let $(M, J, g)$ be an almost Hermitian manifold. For a real-valued smooth function $\varphi$ on $M$, one has

$$
|\partial \partial \bar{\partial} \varphi|_{g} \leq C|Z(\varphi)|_{g}, \quad|\bar{\partial} \partial \bar{\partial} \varphi|_{g} \leq C|Z(\varphi)|_{g}
$$

for a positive constant $C$ which depends on the almost complex structure $J$ and the torsion of the Chern connection.

This paper is organized as follows: in Section 2, we recall some basic definitions and computations on an almost Hermitian manifold $(M, J, g)$. In Section 3, for an arbitrary chosen smooth function $\varphi$ on $M$, we show that $\partial \partial \bar{\partial} \varphi$ and $\bar{\partial} \partial \bar{\partial} \varphi$ depend only on $Z(\varphi), \bar{Z}(\varphi)$ and some geometric quantities of $(M, J, g)$. Notice that we assume the Einstein convention omitting the symbol of sum over repeated indexes in all this paper.

\section{Preliminaries}

\subsection{The Nijenhuis tensor of the almost complex structure}

Let $M$ be a $2 n$-dimensional smooth differentiable manifold. An almost complex structure on $M$ is an endomorphism $J$ the tangent bundle of $T M, J \in \Gamma(\operatorname{End}(T M))$, satisfying $J^{2}=-I d_{T M}$. The pair $(M, J)$ is called an almost complex manifold. Let $(M, J)$ be an almost complex manifold. We define a bilinear map on $C^{\infty}(M)$ for

^Corresponding Author: Masaya Kawamura: Department of General Education National Institute of Technology, Kagawa College 355, Chokushi-cho, Takamatsu, Kagawa, Japan 761-8058, E-mail: kawamura-m@t.kagawa-nct.ac.jp 
$X, Y \in \Gamma(T M)$ by

$$
4 N(X, Y):=[J X, J Y]-J[J X, Y]-J[X, J Y]-[X, Y],
$$

which is the Nijenhuis tensor of $J$. The Nijenhuis tensor $N$ satisfies $N(X, Y)=-N(Y, X), N(J X, Y)=-J N(X, Y)$, $N(X, J Y)=-J N(X, Y), N(J X, J Y)=-N(X, Y)$. For any $(1,0)$-vector fields $V$ and $W, N(V, W)=-[V, W]^{(0,1)}$, $N(V, \bar{W})=N(\bar{V}, W)=0$ and $N(\bar{V}, \bar{W})=-[\bar{V}, \bar{W}]^{(1,0)}$. An almost complex structure $J$ is integrable if $N=0$ on $M$ (Newlander-Nirenberg theorem). Giving a complex structure to a differentiable manifold $M$ is equivalent to giving an integrable almost complex structure to $M$. A Riemannian metric $g$ on $M$ is called $J$-invariant if $J$ is compatible with $g$, i.e., for any $X, Y \in \Gamma(T M), g(X, Y)=g(J X, J Y)$. In this case, the pair $(J, g)$ is called an almost Hermitian structure.

Let $\left\{Z_{r}\right\}$ be a local $(1,0)$-frame on $(M, J)$ with an almost Hermitian metric $g$ and let $\left\{\zeta^{r}\right\}$ be a local associated coframe with respect to $\left\{Z_{r}\right\}$, i.e., $\zeta^{i}\left(Z_{j}\right)=\delta_{j}^{i}$ for $i, j=1, \ldots, n$. Since $g$ is almost Hermitian, its components satsfy $g_{i j}=g_{\overline{i j}}=0$ and $g_{i \bar{j}}=g_{\overline{j i}}=\bar{g}_{\bar{i} j}$.

Using these local frame $\left\{Z_{r}\right\}$ and coframe $\left\{\zeta^{r}\right\}$, we have

$$
N\left(Z_{\bar{i}}, Z_{\bar{j}}\right)=-\left[Z_{\bar{i}}, Z_{\bar{j}}\right]^{(1,0)}=: N_{\bar{i} j}^{k} Z_{k}, \quad N\left(Z_{i}, Z_{j}\right)=-\left[Z_{i}, Z_{j}\right]^{(0,1)}=\overline{N_{\bar{i} j}^{k}} Z_{\bar{k}},
$$

and

$$
N=\frac{1}{2} \overline{N_{\bar{i} j}^{k}} Z_{\bar{k}} \otimes\left(\zeta^{i} \wedge \zeta^{j}\right)+\frac{1}{2} N_{\bar{i} j}^{k} Z_{k} \otimes\left(\zeta^{\bar{i}} \wedge \zeta^{\bar{j}}\right) .
$$

We write $T^{\mathbb{R}} M$ for the real tangent space of $M$. Then its complexified tangent space is given by $T^{\mathbb{C}} M=$ $T^{\mathbb{R}} M \otimes_{\mathbb{R}} \mathbb{C}$. By extending $J \mathbb{C}$-linearly and $g, \mathbb{C}$-bilinearly to $T^{\mathbb{C}} M$, they are also defined on $T^{\mathbb{C}} M$ and we observe that the complexified tangent space $T^{\mathbb{C}} M$ can be decomposed as $T^{\mathbb{C}} M=T^{1,0} M \oplus T^{0,1} M$, where $T^{1,0} M, T^{0,1} M$ are the eigenspaces of $J$ corresponding to eigenvalues $\sqrt{-1}$ and $-\sqrt{-1}$, respectively:

$$
T^{1,0} M=\{X-\sqrt{-1} J X \mid X \in T M\}, \quad T^{0,1} M=\{X+\sqrt{-1} J X \mid X \in T M\} .
$$

Let $\Lambda^{r} M=\bigoplus_{p+q=r} \Lambda^{p, q} M$ for $0 \leq r \leq 2 n$ denote the decomposition of complex differential $r$-forms into $(p, q)$-forms, where $\Lambda^{p, q} M=\Lambda^{p}\left(\Lambda^{1,0} M\right) \otimes \Lambda^{q}\left(\Lambda^{0,1} M\right)$,

$$
\Lambda^{1,0} M=\left\{\eta+\sqrt{-1} J \eta \mid \eta \in \Lambda^{1} M\right\}, \quad \Lambda^{0,1} M=\left\{\eta-\sqrt{-1} J \eta \mid \eta \in \Lambda^{1} M\right\}
$$

and $\Lambda^{1} M$ denotes the dual of $T M$.

Let $(M, J, g)$ be an almost Hermitian manifold with $\operatorname{dim}_{\mathbb{R}} M=2 n$. An affine connection $\nabla$ on $T M$ is called almost Hermitian connection if $D g=D J=0$. For the almost Hermitian connection, we have the following Lemma (cf. [1], [3]).

Lemma 2.1. Let $(M, J, g)$ be an almost Hermitian manifold with $\operatorname{dim}_{\mathbb{R}} M=2 n$. Then for any given complex vector valued $(1,1)$-form $\Theta=\left(\Theta^{i}\right)_{1 \leq i \leq n}$, there exists a unique almost Hermitian connection $\nabla$ on $(M, J, g) \operatorname{such}$ that the $(1,1)$-part of the torsion is equal to the given $\Theta$.

If the $(1,1)$-part of the torsion of an almost Hermitian connection vanishes everywhere, then the connction is called the second canonical connection or the Chern connection. We will refer the connection as the Chern connection and denote it by $\nabla$. Now let $\nabla$ be the Chern connection on $M$. We denote the structure coefficients of Lie bracket by

$$
\left[Z_{i}, Z_{j}\right]=B_{i j}^{r} Z_{r}+B_{i j}^{\bar{r}} Z_{\bar{r}}, \quad\left[Z_{i}, Z_{\bar{j}}\right]=B_{i j}^{r} Z_{r}+B_{i \bar{j}}^{\bar{r}} Z_{\bar{r}}, \quad\left[Z_{\bar{i}}, Z_{\bar{j}}\right]=B_{\bar{i} j}^{r} Z_{r}+B_{\bar{i} j}^{\bar{r}} Z_{\bar{r}} .
$$

We have $B_{i j}^{k}=-B_{j i}^{k}$ since $\left[Z_{i}, Z_{j}\right]=-\left[Z_{j}, Z_{i}\right]$. Notice that $J$ is integrable if and only if the $B_{i j}^{\bar{r}}$, sanish.

For any $p$-form $\psi$, there holds that

$$
\begin{aligned}
d \psi\left(X_{1}, \ldots, X_{p+1}\right)= & \sum_{i=1}^{p+1}(-1)^{i+1} X_{i}\left(\psi\left(X_{1}, \ldots, \widehat{X}_{i}, \ldots, X_{p+1}\right)\right) \\
& +\sum_{i<j}(-1)^{i+j} \psi\left(\left[X_{i}, X_{j}\right], X_{1}, \ldots, \widehat{X}_{i}, \ldots, \widehat{X}_{j}, \ldots, X_{p+1}\right)
\end{aligned}
$$


for any vector fields $X_{1}, \ldots, X_{p+1}$ on $M$ (cf. [3]). We directly compute that

$$
d \zeta^{s}=-\frac{1}{2} B_{k l}^{s} \zeta^{k} \wedge \zeta^{l}-B_{k l}^{s} \zeta^{k} \wedge \zeta^{\bar{l}}-\frac{1}{2} B_{\bar{k} \bar{l}}^{s} \zeta^{\bar{k}} \wedge \zeta^{\bar{l}}
$$

For any real $(1,1)$-form $\eta=\sqrt{-1} \eta_{i j} \zeta^{i} \wedge \zeta^{\bar{j}}$, we have

$$
\begin{aligned}
& \partial \eta=\frac{\sqrt{-1}}{2}\left(Z_{i}\left(\eta_{j \bar{k}}\right)-Z_{j}\left(\eta_{i \bar{k}}\right)-B_{i j}^{s} \eta_{s \bar{k}}-B_{i \bar{k}}^{\bar{s}} \eta_{j \bar{s}}+B_{j \bar{k}}^{\bar{s}} \eta_{i \bar{s}}\right) \zeta^{i} \wedge \zeta^{j} \wedge \zeta^{\bar{k}}, \\
& \bar{\partial} \eta=\frac{\sqrt{-1}}{2}\left(Z_{\bar{j}}\left(\eta_{k \bar{i}}\right)-Z_{\bar{i}}\left(\eta_{k \bar{j}}\right)-B_{k \bar{i}}^{s} \eta_{s \bar{j}}+B_{k \bar{j}}^{s} \eta_{s \bar{i}}+B_{i \bar{j}}^{\bar{s}} \eta_{k \bar{s}}\right) \zeta^{k} \wedge \zeta^{\bar{i}} \wedge \zeta^{\bar{j}}
\end{aligned}
$$

We can split the exterior differential operator $d: \Lambda^{p} M \otimes_{\mathbb{R}} \mathbb{C} \rightarrow \Lambda^{p+1} M \otimes_{\mathbb{R}} \mathbb{C}$, into four components

$$
d=A+\partial+\bar{\partial}+\bar{A}
$$

with

$$
\begin{array}{cl}
\partial: \Lambda^{p, q} M \rightarrow \Lambda^{p+1, q} M, & \bar{\partial}: \Lambda^{p, q} M \rightarrow \Lambda^{p, q+1} M, \\
A: \Lambda^{p, q} M \rightarrow \Lambda^{p+2, q-1} M, & \bar{A}: \Lambda^{p, q} M \rightarrow \Lambda^{p-1, q+2} M .
\end{array}
$$

In terms of these components, the condition $d^{2}=0$ can be written as

$$
\begin{gathered}
A^{2}=0, \quad \partial A+A \partial=0, \bar{\partial} \bar{A}+\bar{A} \bar{\partial}=0, \quad \bar{A}^{2}=0, \\
A \bar{\partial}+\partial^{2}+\bar{\partial} A=0, \quad A \bar{A}+\partial \bar{\partial}+\bar{\partial} \partial+\bar{A} A=0, \quad \partial \bar{A}+\bar{\partial}^{2}+\bar{A} \partial=0 .
\end{gathered}
$$

A direct computation yields for any $\varphi \in C^{\infty}(M, \mathbb{R})$,

$$
(d J d \varphi)\left(Z_{i}, Z_{j}\right)=-2 \sqrt{-1}\left[Z_{i}, Z_{j}\right]^{(0,1)}(\varphi),
$$

by conjugation

$$
(d J d \varphi)\left(Z_{\bar{i}}, Z_{\bar{j}}\right)=2 \sqrt{-1}\left[Z_{\bar{i}}, Z_{\bar{j}}\right]^{(1,0)}(\varphi),
$$

and we have

$$
\begin{aligned}
&(d J d \varphi)\left(Z_{i}, Z_{\bar{j}}\right)=Z_{i}\left(J d \varphi\left(Z_{\bar{j}}\right)\right)-Z_{\bar{j}}\left(J d \varphi\left(Z_{i}\right)\right)-J d \varphi\left(\left[Z_{i}, Z_{\bar{j}}\right]\right) \\
&=-Z_{i}\left(d \varphi\left(J Z_{\bar{j}}\right)\right)+Z_{\bar{j}}\left(d \varphi\left(J Z_{i}\right)\right)+d \varphi\left(J\left[Z_{i}, Z_{\bar{j}}\right]\right) \\
&=\sqrt{-1} Z_{i} Z_{\bar{j}}(\varphi)+\sqrt{-1} Z_{\bar{j}} Z_{i}(\varphi)+J\left[Z_{i}, Z_{\bar{j}}\right](\varphi) \\
&=2 \sqrt{-1} Z_{i} Z_{\bar{j}}(\varphi)-\sqrt{-1}\left(\left[Z_{i}, Z_{\bar{j}}\right]+\sqrt{-1} J\left[Z_{i}, Z_{\bar{j}}\right]\right) \varphi \\
&=2 \sqrt{-1}\left(Z_{i} Z_{\bar{j}}-\left[Z_{i}, Z_{\bar{j}}\right]^{(0,1)}\right) \varphi, \\
& \sqrt{-1} \partial \bar{d} \varphi=\frac{1}{2}(d J d \varphi)^{(1,1)}=\sqrt{-1}\left(Z_{i} Z_{\bar{j}}-\left[Z_{i}, Z_{\bar{j}}\right]^{(0,1)}\right) \varphi \zeta^{i} \wedge \zeta^{\bar{j}},
\end{aligned}
$$

so we write locally

$$
\partial_{i} \partial_{\bar{j}} \varphi=\left(Z_{i} Z_{\bar{j}}-\left[Z_{i}, Z_{\bar{j}}\right]^{(0,1)}\right) \varphi .
$$

\subsection{The torsion and curvature on almost complex manifolds}

Since the Chern connection $\nabla$ preserves $J$, we have

$$
\nabla_{i} Z_{j}=\Gamma_{i j}^{r} Z_{r}, \quad \nabla_{i} Z_{\bar{j}}=\Gamma_{i \bar{j}}^{\bar{r}} Z_{\bar{r}},
$$

where $\Gamma_{i j}^{r}=g^{r \bar{s}} Z_{i}\left(g_{j \bar{s}}\right)-g^{r \bar{s}} g_{j i} \bar{l}_{i \bar{s}}^{\bar{l}}, \Gamma_{i p}^{p}=Z_{i}(\log \operatorname{det} g)-B_{i \bar{s}}^{\bar{s}}$. We can obtain that $\Gamma_{i \bar{j}}^{\bar{r}}=B_{i \bar{j}}^{\bar{r}}$ since the $(1,1)$-part of the torsion of the Chern connection vanishes everywhere. 
Note that the mixed derivatives $\nabla_{i} Z_{\bar{j}}$ do not depend on $g$ (cf. [1]). Let $\left\{\gamma_{j}^{i}\right\}$ be the connection form, which is defined by $\gamma_{j}^{i}=\Gamma_{s j}^{i} \zeta^{s}+\Gamma_{\bar{s} j}^{i} \zeta^{\bar{s}}$. The torsion $T$ of the Chern connection $\nabla$ is given by $T^{i}=d \zeta^{i}-\zeta^{p} \wedge \gamma_{p}^{i}$, $T^{\bar{i}}=d \zeta^{\bar{i}}-\zeta^{\bar{p}} \wedge \gamma_{\bar{p}}^{\bar{i}}$, which has no $(1,1)$-part and the only non-vanishing components are as follows:

$$
T_{i j}^{S}=\Gamma_{i j}^{S}-\Gamma_{j i}^{S}-B_{i j}^{S}=g^{\bar{l} s} Z_{i}\left(g_{j \bar{l}}\right)+g^{\bar{l} s} g_{j \bar{q}} B_{\bar{l}}^{\bar{q}}-g^{\bar{l} s} Z_{j}\left(g_{i \bar{l}}\right)-g^{\bar{l} s} g_{i \bar{q}} B_{\bar{l} j}^{\bar{q}}-B_{i j}^{S} .
$$

We will write the equation above by $B=\Gamma+T^{\prime}$, and we also have $T_{i j}^{\bar{s}}=-B_{i j}^{\bar{s}}$. These tell us that $T$ splits into $T=T^{\prime}+T^{\prime \prime}$, where $T^{\prime} \in \Gamma\left(\Lambda^{2,0} M \otimes T^{1,0} M\right), T^{\prime \prime} \in \Gamma\left(\Lambda^{2,0} M \otimes T^{0,1} M\right)$. We also lower the index of torsion and denote it by

$$
T_{i j \bar{k}}=T_{i j}^{S} g_{s \bar{k}}=Z_{i}\left(g_{j \bar{k}}\right)-Z_{j}\left(g_{i \bar{k}}\right)+B_{\bar{k} i}^{\bar{q}} g_{j \bar{q}}-B_{\bar{k} j}^{\bar{q}} g_{i \bar{q}}-B_{i j}^{S} g_{s \bar{k}} .
$$

Note that $T^{\prime \prime}$ depends only on $J$ and it can be regarded as the Nijenhuis tensor of $J$, that is, $J$ is integrable if and only if $T^{\prime \prime}$ vanishes.

\section{Proof of Theorem 1.1}

Let $(M, J, g)$ be an almost Hermitian manifold. Let $\left\{Z_{r}\right\}$ be an arbitrary local $(1,0)$-frame around a fixed point $p \in M$. Here note that $B_{j \bar{b}}^{\bar{q}}, B_{\bar{j} b}^{q}$ 's do not depend on $g$, which depend only on $J$ since the mixed derivatives $\nabla_{j} Z_{\bar{b}}, \nabla_{\bar{j}} Z_{b}$ do not depend on $g$. Since we have $B_{b \bar{j}}^{q}=-B_{\bar{j} b}^{q}$, we have that $B_{b \bar{j}}^{q}, B_{\bar{b} j}^{\bar{q}}$, s also do not depend on $g$ (cf. [1]). Also note that $B_{r i}^{\bar{s}}, B_{\bar{r} \bar{i}}^{S}$ do not depend on $g$, depend only on $J$. These components $B_{j \bar{b}}^{\bar{q}}, B_{\bar{j} b}^{q}, B_{b \bar{j}}^{q}, B_{\bar{b} j}^{\bar{q}}$, $B_{r i}^{\bar{S}}$ and $B_{\bar{r} \bar{i}}^{S}$ shall be denoted by $B^{\circ}$ in what follows.

Lemma 3.1. One has for a real-valued smooth function $\varphi$ on $M$,

$$
\partial \partial \bar{\partial} \varphi\left(Z_{k}, Z_{j}, Z_{\bar{i}}\right)=B_{k j}^{\bar{S}} Z_{\bar{s}} Z_{\bar{i}}(\varphi)-Z_{k}\left(B_{j \bar{i}}^{\bar{s}}\right) \varphi_{\bar{s}}+Z_{j}\left(B_{k \bar{i}}^{\bar{S}}\right) \varphi_{\bar{s}}+B_{k j}^{S} B_{s \bar{i}}^{\bar{r}} \varphi_{\bar{r}}+B_{k \bar{i}}^{\bar{s}} B_{j \bar{s}}^{\bar{r}} \varphi_{\bar{r}}-B_{j \bar{i}}^{\bar{s}} B_{k \bar{s}}^{\bar{r}} \varphi_{\bar{r}} .
$$

Proof. We compute that from (2.7),

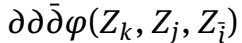

$$
\begin{aligned}
& =Z_{k}\left(\varphi_{j \bar{i}}\right)-Z_{j}\left(\varphi_{k \bar{i}}\right)-B_{k j}^{s} \varphi_{s \bar{i}}-B_{k \bar{s}}^{\bar{s}} \varphi_{j \bar{s}}+B_{j \bar{i}}^{\bar{s}} \varphi_{k \bar{s}} \\
& =Z_{k}\left(Z_{j} Z_{\bar{i}}(\varphi)-B_{j \bar{i}}^{\bar{s}} \varphi_{\bar{s}}\right)-Z_{j}\left(Z_{k} Z_{\bar{i}}(\varphi)-B_{k \bar{i}}^{\bar{S}} \varphi_{\bar{s}}\right) \\
& -B_{k j}^{s}\left(Z_{s} Z_{\bar{i}}(\varphi)-B_{s \bar{r}}^{\bar{r}} \varphi_{\bar{r}}\right)-B_{k \bar{i}}^{\bar{S}}\left(Z_{j} Z_{\bar{s}}(\varphi)-B_{j \bar{s}}^{\bar{r}} \varphi_{\bar{r}}\right)+B_{j \bar{i}}^{\bar{s}}\left(Z_{k} Z_{\bar{s}}(\varphi)-B_{k \bar{s}}^{\bar{r}} \varphi_{\bar{r}}\right) \\
& =Z_{k} Z_{j} Z_{\bar{i}}(\varphi)-Z_{k}\left(B_{j i}^{\bar{s}}\right) \varphi_{\bar{s}}-Z_{j} Z_{k} Z_{\bar{i}}(\varphi)+Z_{j}\left(B_{k i}^{\bar{s}}\right) \varphi_{\bar{s}} \\
& -B_{k j}^{S} Z_{s} Z_{\bar{i}}(\varphi)+B_{k j}^{S} B_{s i}^{\bar{r}} \varphi_{\bar{r}}+B_{k \bar{i}}^{\bar{s}} B_{j \bar{s}}^{\bar{r}} \varphi_{\bar{r}}-B_{j i}^{\bar{s}} B_{k \bar{s}}^{\bar{r}} \varphi_{\bar{r}} \\
& =\left[Z_{k}, Z_{j}\right] Z_{\bar{i}}(\varphi)-B_{k j}^{S} Z_{s} Z_{\bar{i}}(\varphi)-Z_{k}\left(B_{j i}^{\bar{S}}\right) \varphi_{\bar{s}}+Z_{j}\left(B_{k \bar{i}}^{\bar{S}}\right) \varphi_{\bar{s}} \\
& +B_{k j}^{S} B_{s \bar{i}}^{\bar{r}} \varphi_{\bar{r}}+B_{k i}^{\bar{s}} B_{j \bar{s}}^{\bar{r}} \varphi_{\bar{r}}-B_{j i}^{\bar{s}} B_{k \bar{s}}^{\bar{r}} \varphi_{\bar{r}} \\
& =B_{k j}^{\bar{s}} Z_{\bar{s}} Z_{\bar{i}}(\varphi)-Z_{k}\left(B_{j \bar{i}}^{\bar{s}}\right) \varphi_{\bar{s}}+Z_{j}\left(B_{k \bar{i}}^{\bar{s}}\right) \varphi_{\bar{s}}+B_{k j}^{s} B_{\bar{s}}^{\bar{r}} \varphi_{\bar{r}}+B_{k \bar{i}}^{\bar{s}} B_{j \bar{s}}^{\bar{r}} \varphi_{\bar{r}}-B_{j i}^{\bar{s}} B_{k \bar{s}}^{\bar{r}} \varphi_{\bar{r}} .
\end{aligned}
$$

Lemma 3.2. One has for a real-valued smooth function $\varphi$ on $M$,

$$
\begin{aligned}
\bar{\partial} \partial \bar{\partial} \varphi\left(Z_{\bar{k}}, Z_{i}, Z_{\bar{j}}\right)= & B_{\bar{k} \bar{j}}^{S} Z_{i} Z_{s}(\varphi)+Z_{i}\left(B_{\bar{k} \bar{j}}^{S}\right) \varphi_{s}+Z_{i}\left(B_{\bar{k} \bar{j}}^{\bar{s}}\right) \varphi_{\bar{s}}-Z_{\bar{k}}\left(B_{i \bar{j}}^{\bar{s}}\right) \varphi_{\bar{s}}+Z_{\bar{j}}\left(B_{i \bar{k}}^{\bar{s}}\right) \varphi_{\bar{s}} \\
& -B_{i \bar{j}}^{\bar{s}} B_{\bar{k} \bar{s}}^{r} \varphi_{r}-B_{i \bar{j}}^{\bar{s}} B_{\bar{k} \bar{s}}^{\bar{r}} \varphi_{\bar{r}}+B_{i \bar{k}}^{\bar{s}} B_{\bar{j} \bar{s}}^{r} \varphi_{r}+B_{i \bar{k}}^{\bar{s}} B_{\bar{j} \bar{s}}^{\bar{r}} \varphi_{\bar{r}} \\
& +B_{i \bar{j}}^{S} B_{s \bar{k}}^{\bar{r}} \varphi_{\bar{r}}-B_{i \bar{k}}^{S} B_{s \bar{j}}^{\bar{r}} \varphi_{\bar{r}}-B_{\bar{j} \bar{k}}^{\bar{s}} B_{i \bar{s}}^{\bar{r}} \varphi_{\bar{r}} .
\end{aligned}
$$


Proof. We compute from (2.8),

$$
\begin{aligned}
& \bar{\partial} \partial \bar{\partial} \varphi\left(Z_{\bar{k}}, Z_{i}, Z_{\bar{j}}\right) \\
& =Z_{\bar{k}}\left(\varphi_{i \bar{j}}\right)-Z_{\bar{j}}\left(\varphi_{i \bar{k}}\right)-B_{i \bar{j}}^{S} \varphi_{s \bar{k}}+B_{i \bar{k}}^{S} \varphi_{s \bar{j}}+B_{\bar{j} \bar{k}}^{\bar{s}} \varphi_{i \bar{s}} \\
& =Z_{\bar{k}} Z_{i} Z_{\bar{j}}(\varphi)-Z_{\bar{j}} Z_{i} Z_{\bar{k}}(\varphi) \\
& -Z_{\bar{k}}\left(B_{i j}^{\bar{s}}\right) \varphi_{\bar{s}}-B_{i j}^{\bar{s}}\left(Z_{\bar{s}} Z_{\bar{k}} \varphi+\left[Z_{\bar{k}}, Z_{\bar{s}}\right](\varphi)\right)+Z_{\bar{j}}\left(B_{i \bar{k}}^{\bar{s}}\right) \varphi_{\bar{s}}+B_{i \bar{k}}^{\bar{s}}\left(Z_{\bar{s}} Z_{\bar{j}} \varphi+\left[Z_{\bar{j}}, Z_{\bar{s}}\right](\varphi)\right) \\
& -B_{i \bar{j}}^{s}\left(Z_{s} Z_{\bar{k}}(\varphi)-B_{s \bar{k}}^{\bar{r}} \varphi_{\bar{r}}\right)+B_{i \bar{k}}^{s}\left(Z_{s} Z_{\bar{j}}(\varphi)-B_{s \bar{r}}^{\bar{r}} \varphi_{\bar{r}}\right)+B_{\bar{j} \bar{k}}^{\bar{s}}\left(Z_{i} Z_{\bar{s}}(\varphi)-B_{i \bar{s}}^{\bar{r}} \varphi_{\bar{r}}\right) \\
& =Z_{i} Z_{\bar{k}} Z_{\bar{j}}(\varphi)+\left[Z_{\bar{k}}, Z_{i}\right] Z_{\bar{j}}(\varphi)-Z_{i} Z_{\bar{j}} Z_{\bar{k}}(\varphi)-\left[Z_{\bar{j}}, Z_{i}\right] Z_{\bar{k}}(\varphi) \\
& -Z_{\bar{k}}\left(B_{i j}^{\bar{s}}\right) \varphi_{\bar{s}}+Z_{\bar{j}}\left(B_{i \bar{k}}^{\bar{s}}\right) \varphi_{\bar{s}}-\left[Z_{i}, Z_{\bar{j}}\right] Z_{\bar{k}}(\varphi)+\left[Z_{i}, Z_{\bar{k}}\right] Z_{\bar{j}}(\varphi) \\
& -B_{i j}^{\bar{s}}\left[Z_{\bar{k}}, Z_{\bar{s}}\right](\varphi)+B_{i \bar{k}}^{\bar{s}}\left[Z_{\bar{j}}, Z_{\bar{s}}\right](\varphi)+B_{i j}^{s} B_{s \bar{k}}^{\bar{r}} \varphi_{\bar{r}}-B_{i \bar{k}}^{s} B_{s \bar{j}}^{\bar{r}} \varphi_{\bar{r}}-B_{\bar{j} \bar{k}}^{\bar{s}} B_{i \bar{s}}^{\bar{r}} \varphi_{\bar{r}}-B_{\bar{k} \bar{s}}^{\bar{s}} Z_{i} Z_{\bar{s}}(\varphi) \\
& =B_{\bar{k} \bar{j}}^{S} Z_{i} Z_{s}(\varphi)+Z_{i}\left(B_{\bar{k} \bar{j}}^{S}\right) \varphi_{s}+Z_{i}\left(B_{\bar{k} \bar{j}}^{\bar{s}}\right) \varphi_{\bar{s}}-Z_{\bar{k}}\left(B_{i j}^{\bar{s}}\right) \varphi_{\bar{s}}+Z_{\bar{j}}\left(B_{i \bar{k}}^{\bar{s}}\right) \varphi_{\bar{s}} \\
& -B_{i \bar{j}}^{\bar{s}} B_{\bar{k} \bar{s}}^{r} \varphi_{r}-B_{i \bar{j}}^{\bar{s}} B_{\bar{k} \bar{s}}^{\bar{r}} \varphi_{\bar{r}}+B_{i \bar{k}}^{\bar{s}} B_{\bar{j} \bar{s}}^{r} \varphi_{r}+B_{i \bar{k}}^{\bar{s}} B_{\bar{j} \bar{s}}^{\bar{r}} \varphi_{\bar{r}}+B_{i \bar{j}}^{s} B_{s \bar{k}}^{\bar{r}} \varphi_{\bar{r}}-B_{i \bar{k}}^{S} B_{s \bar{j}}^{\bar{r}} \varphi_{\bar{r}}-B_{\bar{j} \bar{k}}^{\bar{s}} B_{i \bar{s}}^{\bar{r}} \varphi_{\bar{r}},
\end{aligned}
$$

where we have used that $B_{\bar{k} \bar{j}}^{\bar{s}}=-B_{\bar{j} \bar{k}}^{\bar{s}}$,

$$
\left[Z_{i}, Z_{\bar{j}}\right] Z_{\bar{k}}(\varphi)=B_{i \bar{j}}^{\bar{s}} Z_{\bar{s}} Z_{\bar{k}}(\varphi)+B_{i \bar{j}}^{s} Z_{s} Z_{\bar{k}}(\varphi), \quad\left[Z_{i}, Z_{\bar{k}}\right] Z_{\bar{j}}(\varphi)=B_{i \bar{k}}^{\bar{s}} Z_{\bar{s}} Z_{\bar{j}}(\varphi)+B_{i \bar{k}}^{s} Z_{s} Z_{\bar{j}}(\varphi)
$$

and that

$$
\begin{aligned}
Z_{i} Z_{\bar{k}} Z_{\bar{j}}(\varphi)-Z_{i} Z_{\bar{j}} Z_{\bar{k}}(\varphi) & =Z_{i}\left[Z_{\bar{k}}, Z_{\bar{j}}\right](\varphi) \\
& =Z_{i}\left(B_{\bar{k} \bar{j}}^{S} Z_{s}+B_{\bar{k} \bar{j}}^{\bar{s}} Z_{\bar{s}}\right)(\varphi) \\
& =Z_{i}\left(B_{\bar{k} j}^{S}\right) \varphi_{s}+B_{\bar{k} \bar{j}}^{S} Z_{i} Z_{s}(\varphi)+Z_{i}\left(B_{\bar{k} j}^{\bar{s}}\right) \varphi_{\bar{s}}+B_{\bar{k} j}^{\bar{s}} Z_{i} Z_{\bar{s}}(\varphi) .
\end{aligned}
$$

Since we have

$$
\begin{aligned}
\bar{\partial} \partial \bar{\partial} \varphi\left(Z_{\bar{k}}, Z_{i}, Z_{\bar{j}}\right) & =\overline{\partial \bar{\partial} \partial \varphi\left(Z_{k}, Z_{\bar{i}}, Z_{j}\right)} \\
& =-\overline{\partial \partial \bar{\partial} \varphi\left(Z_{k}, Z_{j}, Z_{\bar{i}}\right)},
\end{aligned}
$$

where we have used that $\bar{\partial} \partial \varphi=-(\partial \bar{\partial}+A \bar{A}+\bar{A} A) \varphi=-\partial \bar{\partial} \varphi$ since $A \varphi=\bar{A} \varphi=0$, we obtain that by combining (3.1) and (3.2) with (3.3),

$$
\begin{aligned}
& B_{\bar{k} \bar{j}}^{S} Z_{i} Z_{s}(\varphi)+Z_{i}\left(B_{\bar{k} \bar{j}}^{S}\right) \varphi_{s}+Z_{i}\left(B_{\bar{k} \bar{j}}^{\bar{s}}\right) \varphi_{\bar{s}}-Z_{\bar{k}}\left(B_{i \bar{j}}^{\bar{S}}\right) \varphi_{\bar{s}}+Z_{\bar{j}}\left(B_{i \bar{k}}^{\bar{S}}\right) \varphi_{\bar{s}} \\
& -B_{i \bar{j}}^{\bar{s}} B_{\bar{k} \bar{s}}^{r} \varphi_{r}-B_{i \bar{j}}^{\bar{s}} B_{\bar{k} \bar{s}}^{\bar{r}} \varphi_{\bar{r}}+B_{i \bar{k}}^{\bar{s}} B_{\bar{j} \bar{s}}^{r} \varphi_{r}+B_{i \bar{k}}^{\bar{s}} B_{\bar{j} \bar{s}}^{\bar{r}} \varphi_{\bar{r}}+B_{i \bar{j}}^{s} \bar{s}_{s \bar{k}}^{\bar{r}} \varphi_{\bar{r}}-B_{i \bar{k}}^{S} B_{s \bar{j}}^{\bar{r}} \varphi_{\bar{r}}-B_{\bar{j} \bar{k}}^{\bar{s}} B_{i \bar{s}}^{\bar{r}} \varphi_{\bar{r}} \\
& =-B_{\bar{k} j}^{S} Z_{s} Z_{i}(\varphi)+Z_{\bar{k}}\left(B_{\bar{j} i}^{S}\right) \varphi_{s}-Z_{\bar{j}}\left(B_{\bar{k} i}^{S}\right) \varphi_{s}-B_{\bar{k} \bar{j}}^{\bar{S}} B_{\bar{s} i}^{r} \varphi_{r}-B_{\bar{k} i}^{S} B_{\bar{j} s}^{r} \varphi_{r}+B_{j i}^{S} B_{\bar{k} s}^{r} \varphi_{r} \\
& =-B_{\bar{k} j}^{S} Z_{i} Z_{s}(\varphi)-B_{\bar{k} \bar{j}}^{S}\left[Z_{s}, Z_{i}\right](\varphi)+Z_{\bar{k}}\left(B_{j i}^{S}\right) \varphi_{s}-Z_{\bar{j}}\left(B_{\bar{k} i}^{S}\right) \varphi_{s} \\
& -B_{\bar{k} j}^{\bar{s}} B_{\bar{s} i}^{r} \varphi_{r}-B_{\bar{k} i}^{s} B_{j s}^{r} \varphi_{r}+B_{j i}^{s} B_{\bar{k} s}^{r} \varphi_{r} \\
& =-B_{\bar{k} j}^{S} Z_{i} Z_{s}(\varphi)+Z_{\bar{k}}\left(B_{\bar{j} i}^{S}\right) \varphi_{s}-Z_{\bar{j}}\left(B_{\bar{k} i}^{S}\right) \varphi_{s} \\
& -B_{\bar{k} \bar{j}}^{S} B_{s i}^{r} \varphi_{r}-B_{\bar{k} \bar{j}}^{S} B_{s i}^{\bar{r}} \varphi_{\bar{r}}-B_{\bar{k} j}^{\bar{s}} B_{\bar{s} i}^{r} \varphi_{r}-B_{\bar{k} i}^{S} B_{\bar{j} s}^{r} \varphi_{r}+B_{\bar{j} i}^{S} B_{\bar{k} s}^{r} \varphi_{r} .
\end{aligned}
$$

Hence we have the following Lemma.

Lemma 3.3. One has for a real-valued smooth function $\varphi$ on $M$,

$$
\begin{aligned}
& 2 B_{\bar{k} j}^{S} Z_{i} Z_{S}(\varphi) \\
& =-Z_{i}\left(B_{\bar{k} \bar{j}}^{S}\right) \varphi_{s}-Z_{i}\left(B_{\bar{k} \bar{j}}^{\bar{S}}\right) \varphi_{\bar{s}}+Z_{\bar{k}}\left(B_{i \bar{j}}^{\bar{S}}\right) \varphi_{\bar{s}}-Z_{\bar{j}}\left(B_{i \bar{k}}^{\bar{S}}\right) \varphi_{\bar{s}}+Z_{\bar{k}}\left(B_{\bar{j} i}^{S}\right) \varphi_{s}-Z_{\bar{j}}\left(B_{\bar{k} k}^{S}\right) \varphi_{s} \\
& +B_{i \bar{j}}^{\bar{s}} B_{\bar{k} \bar{s}}^{r} \varphi_{r}+B_{i \bar{j}}^{\bar{s}} B_{\bar{k} \bar{s}}^{\bar{r}} \varphi_{\bar{r}}-B_{i \bar{k}}^{\bar{s}} B_{\bar{j} \bar{s}}^{r} \varphi_{r}-B_{i \bar{k}}^{\bar{s}} B_{\bar{j} \bar{s}}^{\bar{r}} \varphi_{\bar{r}}-B_{i \bar{j}}^{s} \bar{S}_{s \bar{k}}^{\bar{r}} \varphi_{\bar{r}}+B_{i \bar{k}}^{s} B_{s \bar{j}}^{\bar{r}} \varphi_{\bar{r}}+B_{\bar{j} \bar{k}}^{\bar{s}} B_{i \bar{s}}^{\bar{r}} \varphi_{\bar{r}} \\
& -B_{\bar{k} j}^{S} B_{s i}^{r} \varphi_{r}-B_{\bar{k} j}^{S} B_{s i}^{\bar{r}} \varphi_{\bar{r}}-B_{\bar{k} j}^{\bar{S}} B_{\bar{s} i}^{r} \varphi_{r}-B_{\bar{k} i}^{S} B_{\bar{j} s}^{r} \varphi_{r}+B_{j i}^{S} B_{\bar{k} s}^{r} \varphi_{r}
\end{aligned}
$$


Proof of Theorem 1.1. In this proof, in order to avoid a notational quagmire, we adopt the following *convention $A_{1} \star A_{2}$ between two quantities $A_{1}$ and $A_{2}$ with respect to a metric $g$ :

(1) Summation over pairs of maching upper and lower indices.

(2) Contraction on upper indices with respect to the metric.

(3) Contraction on lower indices with respect to the dual metrics.

Let $\left\{Z_{r}\right\}$ be a local $(1,0)$-frame with respect to $g$ around a fixed point $p \in M$ (we call it a local $g$-unitary frame in the following) and let $\left\{\zeta^{r}\right\}$ be a local associated coframe with respect to $\left\{Z_{r}\right\}$, i.e., $\zeta^{i}\left(Z_{j}\right)=\delta_{j}^{i}$ for $i, j=1, \ldots, n$. Note that unitary frames always exist locally since we can take any frame and apply the GramSchmidt process. Then with respect to such a frame, we have $g_{i j}=\delta_{i j}, Z_{k}\left(g_{i j}\right)=0$ for any $i, j, k=1, \ldots, n$, and the Christoffel symbols satisfy

$$
\Gamma_{i j}^{k}=-\Gamma_{i \bar{k}}^{\bar{j}}=-B_{i \bar{k}}^{\bar{j}}
$$

since we compute that

$$
\Gamma_{i j}^{k}=g\left(\nabla_{i} Z_{j}, Z_{\bar{k}}\right)=Z_{i}\left(g_{j \bar{k}}\right)-g\left(Z_{j}, \nabla_{i} Z_{\bar{k}}\right)=-\Gamma_{i \bar{k}}^{\bar{j}} .
$$

Fix a local $g$-unitary frame $\left\{Z_{r}\right\}$ in this proof. We choose a smooth function $\varphi$ arbitrary. Then we have the following formula:

$$
\begin{aligned}
& 2 B_{\bar{k} \bar{j}}^{S} Z_{i} Z_{s}(\varphi) \\
& =-Z_{i}\left(B_{\bar{k} \bar{s}}^{S}\right) \varphi_{s}+Z_{i}\left(T_{\bar{k} j}^{\bar{s}}\right) \varphi_{\bar{s}}+Z_{i}\left(B_{\bar{k} s}^{j}\right) \varphi_{\bar{s}}-Z_{i}\left(B_{\bar{j} s}^{k}\right) \varphi_{\bar{s}}+Z_{\bar{k}}\left(B_{i j}^{\bar{s}}\right) \varphi_{\bar{s}}-Z_{\bar{j}}\left(B_{i \bar{k}}^{\bar{s}}\right) \varphi_{\bar{s}} \\
& +Z_{\bar{k}}\left(B_{\bar{j}}^{S}\right) \varphi_{s}-Z_{\bar{j}}\left(B_{\bar{k} i}^{S}\right) \varphi_{s}+B_{i \bar{j}}^{\bar{S}} B_{\bar{k} \bar{s}}^{r} \varphi_{r}-B_{i \bar{j}}^{\bar{s}} T_{\bar{k} \bar{s}}^{\bar{r}} \varphi_{\bar{r}}-B_{i \bar{j}}^{\bar{s}} B_{\bar{k} r}^{S} \varphi_{\bar{r}}+B_{i j}^{\bar{s}} B_{\bar{s} r}^{k} \varphi_{\bar{r}} \\
& -B_{i \bar{k}}^{\bar{S}} B_{\bar{j} \bar{s}}^{r} \varphi_{r}+B_{i \bar{k}}^{\bar{S}} T_{\bar{j} \bar{s}}^{\bar{r}} \varphi_{\bar{r}}+B_{i \bar{k}}^{\bar{S}} B_{j r}^{S} \varphi_{\bar{r}}-B_{i \bar{k}}^{\bar{s}} B_{\bar{s} r}^{j} \varphi_{\bar{r}}-B_{i \bar{j}}^{S} B_{s \bar{k}}^{\bar{r}} \varphi_{\bar{r}}+B_{i \bar{k}}^{S} B_{s \bar{j}}^{\bar{r}} \varphi_{\bar{r}} \\
& -T_{\bar{j} \bar{k}}^{\bar{s}} B_{i \bar{s}}^{\bar{r}} \varphi_{\bar{r}}-B_{\bar{j} s}^{k} B_{i \bar{s}}^{\bar{r}} \varphi_{\bar{r}}+B_{\bar{k} s}^{j} B_{i \bar{s}}^{\bar{r}} \varphi_{\bar{r}}+B_{\bar{k} \bar{j}}^{S} T_{s i}^{r} \varphi_{r}+B_{\bar{k} \bar{s}}^{S} B_{s \bar{r}}^{\bar{i}} \varphi_{r}-B_{\bar{k} \bar{j}}^{S} B_{i \bar{r}}^{\bar{s}} \varphi_{r} \\
& -B_{\bar{k} j}^{S} B_{s i}^{\bar{r}} \varphi_{\bar{r}}+T_{\bar{k} j}^{\bar{s}} B_{\bar{s} i}^{r} \varphi_{r}+B_{\bar{k} s}^{j} B_{\overline{s i}}^{r} \varphi_{r}-B_{\bar{j} s}^{k} B_{\bar{s} i}^{r} \varphi_{r}-B_{\bar{k} i}^{S} B_{\bar{j} s}^{r} \varphi_{r}+B_{j i}^{S} B_{\bar{k} s}^{r} \varphi_{r} \\
& =Z\left(B^{\circ}\right) \star Z(\varphi)+Z\left(\bar{T}^{\prime}\right) \star \bar{Z}(\varphi)+Z\left(B^{\circ}\right) \star \bar{Z}(\varphi)+\bar{Z}\left(B^{\circ}\right) \star \bar{Z}(\varphi)+\bar{Z}\left(B^{\circ}\right) \star Z(\varphi) \\
& +B^{\circ} \star B^{\circ} \star \bar{Z}(\varphi)+B^{\circ}{ }^{\circ}{ }^{\circ} Z(\varphi)+B^{\circ} \star \bar{T}^{\prime} \star \bar{Z}(\varphi)+B^{\circ}{ }^{\prime} T^{\star} Z(\varphi)+\bar{T}^{\prime} \star B^{\circ} Z(\varphi) \text {, }
\end{aligned}
$$

where we used that $T_{i j}^{k}=\Gamma_{i j}^{k}-\Gamma_{j i}^{k}-B_{i j}^{k}$ and $\Gamma_{i j}^{k}=-\Gamma_{i \bar{k}}^{\bar{j}}=-B_{i \bar{k}}^{\bar{j}}$.

Then we have the following formula by applying Lemma 3.3, by conjugation of $2 B_{\bar{k} \bar{j}}^{s} Z_{i} Z_{s}(\varphi)$,

$$
\begin{aligned}
& 2 B_{k j}^{\bar{s}} Z_{\bar{s}} Z_{\bar{i}}(\varphi) \\
= & 2 B_{k j}^{\bar{s}} B_{\bar{s} \bar{i}}^{\bar{r}} \varphi_{\bar{r}}+2 B_{k j}^{\bar{s}} B_{\bar{s} \bar{i}}^{r} \varphi_{r}+2 \overline{B_{\bar{k} \bar{j}}^{s} Z_{i} Z_{s}(\varphi)} \\
= & \bar{Z}\left(B^{\circ}\right) \star \bar{Z}(\varphi)+\bar{Z}\left(T^{\prime}\right) \star Z(\varphi)+\bar{Z}\left(B^{\circ}\right) \star Z(\varphi)+Z\left(B^{\circ}\right) \star Z(\varphi)+Z\left(B^{\circ}\right) \star \bar{Z}(\varphi) \\
& +B^{\circ \star} B^{\circ \star} Z(\varphi)+B^{\circ \star} B^{\circ} \star \bar{Z}(\varphi) \\
& +B^{\circ \star} T^{\prime \star} Z(\varphi)+B^{\circ \star} \bar{T}^{\prime} \star \bar{Z}(\varphi)+T^{\prime} \star B^{\circ} \bar{Z}(\varphi) .
\end{aligned}
$$

By combining (3.4) with (3.1), we obtain that

$$
\begin{aligned}
& \partial \partial \bar{\partial} \varphi\left(Z_{k}, Z_{j}, Z_{\bar{i}}\right) \\
& =B_{k j}^{\bar{s}} Z_{\bar{s}} Z_{\bar{i}}(\varphi)-Z_{k}\left(B_{j i}^{\bar{s}}\right) \varphi_{\bar{s}}+Z_{j}\left(B_{k i}^{\bar{s}}\right) \varphi_{\bar{s}}+B_{k j}^{s} B_{\bar{s} i}^{\bar{r}} \varphi_{\bar{r}}+B_{k i}^{\bar{s}} B_{j \bar{s}}^{\bar{r}} \varphi_{\bar{r}}-B_{j i}^{\bar{s}} B_{k \bar{s}}^{\bar{r}} \varphi_{\bar{r}} \\
& =B_{k j}^{\bar{s}} Z_{\bar{s}} Z_{\bar{i}}(\varphi)-Z_{k}\left(B_{j \bar{i}}^{\bar{s}}\right) \varphi_{\bar{s}}+Z_{j}\left(B_{k \bar{i}}^{\bar{s}}\right) \varphi_{\bar{s}}-T_{k j}^{s} B_{s \bar{i}}^{\bar{r}} \varphi_{\bar{r}} \\
& -B_{k \bar{s}}^{\bar{j}} B_{s \bar{i}}^{\bar{r}} \varphi_{\bar{r}}+B_{j \bar{s}}^{\bar{k}} B_{s i}^{\bar{r}} \varphi_{\bar{r}}+B_{k \bar{i}}^{\bar{s}} B_{j \bar{s}}^{\bar{r}} \varphi_{\bar{r}}-B_{j i}^{\bar{s}} B_{k \bar{s}}^{\bar{r}} \varphi_{\bar{r}} \\
& =\bar{Z}\left(B^{\circ}\right) \star \bar{Z}(\varphi)+\bar{Z}\left(T^{\prime}\right) \star Z(\varphi)+\bar{Z}\left(B^{\circ}\right) \star Z(\varphi)+Z\left(B^{\circ}\right) \star Z(\varphi)+Z\left(B^{\circ}\right) \star \bar{Z}(\varphi) \\
& +B^{\circ} B^{\circ} \star Z(\varphi)+B^{\circ} B^{\circ} \star \bar{Z}(\varphi) \\
& +B^{\circ} \star T^{\prime} \star Z(\varphi)+B^{\circ} \star \bar{T}^{\prime} \star \bar{Z}(\varphi)+T^{\prime} \star B^{\circ} \star \bar{Z}(\varphi) .
\end{aligned}
$$


Then, we have by conjugation of $\partial \partial \bar{\partial} \varphi\left(Z_{k}, Z_{j}, Z_{\bar{i}}\right)$ as in (3.3),

$$
\begin{aligned}
& \bar{\partial} \partial \bar{\partial} \varphi\left(Z_{\bar{k}}, Z_{i}, Z_{\bar{j}}\right) \\
= & Z\left(B^{\circ}\right) \star Z(\varphi)+Z\left(\bar{T}^{\prime}\right) \star \bar{Z}(\varphi)+Z\left(B^{\circ}\right) \star \bar{Z}(\varphi)+\bar{Z}\left(B^{\circ}\right) \star \bar{Z}(\varphi)+\bar{Z}\left(B^{\circ}\right) \star Z(\varphi) \\
& +B^{\circ \star} B^{\circ \star} \bar{Z}(\varphi)+B^{\circ} \star B^{\circ} \star Z(\varphi) \\
& +B^{\circ \star} \bar{T}^{\prime} \star \bar{Z}(\varphi)+B^{\circ \star} T^{\prime} \star Z(\varphi)+\bar{T}^{\prime} \star B^{\circ} \star Z(\varphi) .
\end{aligned}
$$

Remark 3.1. If we assume that $\partial \partial \bar{\partial} \varphi=0$ (resp. $\bar{\partial} \partial \bar{\partial} \varphi=0$ ), since a real-valued smooth function $\varphi$ is chosen arbitrary, the coefficient of the second order term vanishes, that is, we have that $B_{k j}^{\bar{S}}=0$ (resp. $\left.B_{\bar{k} \bar{j}}^{S}=0\right)$, which tells us that the almost complex structure $J$ is then integrable. Note that in the quasi-Kähler case, which includes almost Kähler and nearly Kähler cases, since in these cases we have $T_{i j}^{k}=0$ for all $i, j$ and $k$ (cf. [2]), we have that from (3.5),

$$
\begin{aligned}
\partial \partial \bar{\partial} \varphi\left(Z_{k}, Z_{j}, Z_{\bar{i}}\right)= & \bar{Z}\left(B^{\circ}\right) \star \bar{Z}(\varphi)+\bar{Z}\left(B^{\circ}\right) \star Z(\varphi)+Z\left(B^{\circ}\right) \star Z(\varphi)+Z\left(B^{\circ}\right) \star \bar{Z}(\varphi) \\
& +B^{\circ} B^{\circ} Z^{\circ}(\varphi)+B^{\circ} B^{\circ} \star \bar{Z}(\varphi) .
\end{aligned}
$$

Acknowledgement: This work was supported by JSPS KAKENHI Grant Number JP21K13798.

\section{References}

[1] Vezzoni, L. On Hermitian curvature flow on almost complex manifolds, Diff. Geom. and its Appl. 29 (2011), 709-722.

[2] Yu, C J. Curvature identities on almost Hermitian manifolds and applications, Sci. China Math. 60 (2) (2017), 285-300.

[3] Zheng, T. An almost complex Chern-Ricci flow, J. Geom. Anal. (2017). https://doi.org/10.1007/s12220-017-9898-9. 